\title{
Prevalence of Methicillin-Resistant Staphylococcus aureus in Hospitals of Kathmandu Valley
}

\author{
Reena K. Mukhiya1 ${ }^{1}$, Anima Shrestha ${ }^{1}$, Shiba K. Rai ${ }^{2,3}$, Kritu Panta ${ }^{2}$ R.N. Singh ${ }^{4}$, \\ Ganesh Rai ${ }^{4}$,Amita Prajapati ${ }^{3}$ \\ ${ }^{1}$ St. Xavier's College \\ Maitighar Kathmandu \\ ${ }^{2}$ Shi-Gan Health Foundation, \\ ${ }^{3}$ Nat'l Institute of Tropical Medicine and Public Health Research, \\ Taku, Kathmandu \\ ${ }^{4}$ Nepal Police Hospital \\ MaharajJung, Kathmandu \\ e-mail: aastha_rina@yahoo.com
}

\begin{abstract}
Present study was conducted to determine the prevalence of nosocomial pathogen methicillin resistant Staphylococcus aureus in hospital environment samples (surface swabs and air, $\mathrm{n}=188$ ) and health care workers (nose and hand, $\mathrm{n}=162$ ) of hospitals located in Kathmandu valley. The samples were studied following the standard protocols. Of the 61 S. aureus, $20.9 \%$ and $14.3 \%$ were isolated from health personnel and hospital environment respectively. Of 20.9\% S. aureus in hospital environment, 6.6\% were isolated from OPD and 17.9\% from wards. Similarly, in HCWs 28.3\% and 13.5\% were hand and nasal carrier respectively. In total, 45.9\% were MRSA. Among MRSA isolates, 50\% were from HCWs whereas, $40.7 \%$ from hospital environment. The most effective antibiotic for all isolates was vancomycin with $100 \%$ efficacy. The isolates had relatively high rate of resistance to cefixime (44.0\%), followed by co-trimoxazole (28.0\%), erythromycin (23.0\%), gentamicin (18.0\%), tetracycline (16.0\%) and ofloxacin (8.0\%). Since MRSA prevalence was not reduced, so indicates regular surveillance of nosocomial infection, hand hygiene improvement strategies and monitoring of antimicrobial susceptibility pattern.
\end{abstract}

Key words: healthcare workers, hospital environment, methicillin resistant S. aureus, nosocomial infection

\section{Introduction}

Staphylococcus aureus, a gram positive cocci, is the major cause of both hospital and community acquired infections worldwide (Kluytmans 1998). S. aureus is a common pathogenic commensal bacterium, where approximately $60 \%$ of the human populations are by it and as high as $20 \%$ humans are persistent carriers (Friendship \& Weese 2009).

S. aureus causes a range of infections from superficial abscesses and boils to the more serious infections of osteomyelitis, septicaemia and pneumonia. Approximately, 25\% of all nosocomial infections are caused by $S$. aureus and leading to increase hospital stay, antibiotic use, costs and mortality (Minties-de Groot et al. 2000, Pittet et al. 1995).

The incidence of community-acquired and hospital acquired $S$. aureus infections has been rising with increasing emergence of drug-resistant strains called methicillin resistant S. aureus (MRSA) (Shakya et al. 2010). MRSA is a major cause of morbidity and mortality around the world (Hiramatsu et al. 2001) and has become a challenge to the physicians for its treatment which are often multi drug resistant (Crawcraft \& Catchpole 2002). Hospital environment (surfaces, air) and carrier (nasal and hand) of healthcare workers may serve as reservoir and transmission occurs from HCWs to patients or among the patients (Boyce et al. 1997). 
The percentage of hospitals isolation MRSA in the developed countries has increased from $2 \%$ in the 1970 s to $30 \%$ in the 90 's (Gordon 1993). In UK, $44.0 \%$ of $S$. aureus isolated from health care system are MRSA (Gould 2005) and in Japan 60.0-70.0\% of S. aureus are MRSA in inpatients (Kikuchi 2003). In Nepal, prevalence of MRSA shows an increasing trend; $29.1 \%-68.0 \%$ (Rai et al. 1990, Shrestha et al. 2009, Rijal et al. 2008, Khanal \& Jha 2010). Therefore, objective of this study was to determine prevalence of MRSA.

\section{Methodology}

Sample collection: Hospital environments comprised surface swabs of bed bar, bed sheet, nursing station, cuffs, etc and air samples (taken by plate exposure technique for 20- 25 minutes). Similarly, HCWs comprised swab of palm and web space between fingers of hand (cotton swab dipped in BHI broth) and nasal swab (sterile cotton swab rotated 4-5 minutes clockwise and anticlockwise within nasal cavity). Study design was cross sectional. After proper sampling, the specimens were transported to laboratory of Nat'l Institute of Tropical Medicine and Public Health Research (NITMPHR) and processed immediately.

Sample processing and bacterial identification: Specimens were inoculated into MacConkey Agar (MA), Blood Agar (BA) and Mannitol Salt Agar (MSA) (only for nasal) and incubated at $37^{\circ} \mathrm{C}$ for 24 hours. Gram positive cocci in clusters, catalase +ve, fermentative, manitol fermentor and coagulase +ve were identified as S. aureus.

Antibiotic susceptibility test: All the identified isolates of $S$. aureus were undertaken in-vitro antibiotic susceptibility test by using Kirby Bauer's disc diffusion method. The antibiotics used were Cefixime ( $5 \mathrm{mcg} / \mathrm{disc})$, Methicillin (30mcg/disc), Co-trimoxazole (25mcg/disc), Ofloxacin (5mcg/disc), Tetracycline (30mcg/disc), Gentamycin (10mcg/disc), Erythromycin (15mcg/disc), and Vancomycin (30mcg/disc).

\section{Results and Discussion}

Of the 350 samples studied, S. aureus was isolated from 61 samples (17.4\%). The prevalence rate of $S$. aureus in HCWs were 20.9\% and hospital environment (14.3\%) were found. Out of 61 S. aureus isolates, 28 (45.9\%) were methicillin resistant. Among them, 17(50.0\%) and 11(40.7\%) were from HCWs and hospital environment respectively (Table 1). Similarly, in hospital environment the prevalence rate of S. aureus in OPDs was found to be $6.6 \%$ and $17.9 \%$ in wards (Table 2). In HCWs, $13.5 \%$ and $28.3 \%$ were nasal and hand carrier respectively (Table 3).

Among 61 S. aureus isolates, $45.9 \%$ showed resistant towards methicillin followed by cefixime (44.0\%), cotrimoxazole (28.0\%), erythromycin (23.0\%), gentamicin (18.0\%), tetracycline (16.0\%) and ofloxacin (8.0\%), while none $(0.0 \%)$ were resistant to vancomycin (Fig. 1).

Table 1. Frequency of S. aureus and MRSA from different sample sources

\begin{tabular}{c|c|c|c}
\hline Sample sources & Total samples & S. aureus (\%) & MRSA (\%) \\
\hline Health-personnel & 162 & $34(20.9 \%)$ & $17(50 \%)$ \\
Hospital environment & 188 & $27(14.3 \%)$ & $11(40.7 \%)$ \\
\hline Total & 350 & $61(17.4 \%)$ & $28(45.9 \%)$ \\
\hline
\end{tabular}

Table 2. Frequency of S. aureus from environment samples

\begin{tabular}{l|c|c|c}
\hline \multicolumn{1}{c|}{ Sampling site } & Total sample & +ve & $\%$ \\
\hline OPDs & 60 & 4 & 6.6 \\
Wards & 128 & 23 & 17.9 \\
\hline Total & 188 & 27 & 14.3 \\
\hline
\end{tabular}

Table 3. Frequency of $S$. aureus from nose and hand of HCWs

\begin{tabular}{l|l|l|l}
\hline Sample site & Total sample & +ve & $\%$ \\
\hline Hand & 81 & 23 & 28.3 \\
Nose & 81 & 11 & 13.5 \\
\hline Total & 162 & 34 & 20.9 \\
\hline
\end{tabular}




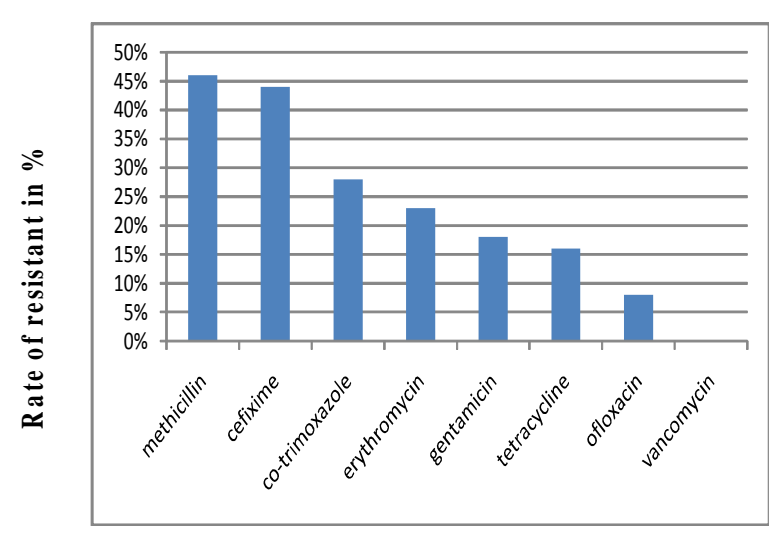

Antibiotics used

Fig. 1. Resistant pattern shown by total S. aureus isolated

MRSA is a global phenomenon with a prevalence rate ranging from $2 \%$ in Netherlands and Switzerland, to $70 \%$ in Japan and Hong Kong (Fluit et al. 2001, Diekema et al. 2001). In this study, the prevalence of MRSA was found to be $45.9 \%$ which was in accordance with the reports by Rijal et al. 2008, Shrestha et al. 2009 and Shakya et al. 2010. On the contrary, some of the reports show an alarmingly high incidence of MRSA (Khanal \& Jha 2010, Ajamal et al. 2009). Higher isolation rates reported in these studies can be attributed to several factors. These include indiscriminate use of antibiotics, lack of awareness and failure to observe simple yet effective infection control precautions like strict patient isolation and frequent hand washing by health care personnel, population and area studied, etc.

Present study revealed that the prevalence of MRSA in hospital environment was $40.7 \%$ which was higher than reports from Panta et al. 2006 and Boyce et al. 1997. This was, however, significantly less as compared to the results on clinical samples (Khanal \& Jha 2010, Shrestha et al. 2009, Verma et al. 2000, Ahmad et al. 2007. The difference might be due to the reason that clinical isolates showed higher degree of resistance to antibiotics than environmental isolates (Shaffer \& Goldin 1969). Similarly present study showed the prevalence of $S$. aureus was high in wards than in OPD. It might be due to large sample size from wards. This report was contrary from Chitwan (Sanjana et al. 2010).

The 28.3\% hand samples showing S. aureus was lower than that has been reported by Zahoor et al, and Pant et al. among hospital staffs. The lower hand carrier rate among the study subjects observed in this study was indicative of adequate hygienic practice.

The nasal carriage rate of $S$. aureus (13.5\%) in the present study was lower than the rate reported (Tejero 1991, Akhtar 2010). Likewise, relatively higher prevalence has been reported in community (Onanuga \& Temedie 2011). It might be due the distribution of $S$. aureus which varies according to factors such as population, area of study, the use of different culture technique and different interpretation guidelines, etc.

Report of this research showed S. aureus was highly resistant towards â-lactam antibiotics like methicillin and cefixime among the others which were in accordance with the findings of Shakya et al. Resistance to quinolones was not high as compared with the reports from Khanal \& Jha (2010) and Kumari et al. (2008). The low resistance rate in our study was probably due to the samples of hospital environment as well as indiscriminate and empirical use of the drugs in other study.

There was low resistance towards gentamicin which was similar with the reports of Onanuga et al. (2005). This may be due to the absence of resistance conferring genes in these MRSA strains as reported by (Polyzou et al. 2001). Vancomycin seems to be the only antimicrobial agent which showed $100 \%$ sensitivity and so may be used as the drug of choice for treating MRSA infections. Vancomycin is not a commonly prescribed drug, which is almost certainly due to its higher price and its unavailability in many parts of the country.

Small number of MRSA was found from the hospital environment and staff in comparison with other study (Khanal \& Jha 2010, McCarthy et al. 2010, Vidhani et al. 2001). Even though, this findings focuses for cleaning, disinfection and sterilization of hospital environment, and regular surveillance of microbial flora among hospital staff and environment to prevent MRSA transmission among them.

\section{Acknowledgements}

We would like to acknowledge Mr. Sudhakar Pant, Mrs Chandra kala Rai and entire staffs of Nepal Police Hospital, Shi-Gan Health Foundation and St. Xavier's College. 
Nepal Journal of Science and Technology Vol. 13, No. 2 (2012) 185-190

\section{References}

Ahmad, S. 2009. Methicillin resistant among clinical isolates of Staphylococcus aureus isolated at a microbiology diagnostic center in Kashmir. Rawal Medical Journal 34: 92-94.

Ajmal A., F. Mir, M. Aslam, R. Hafeez and R. Attique. 2009. Nosocomial methicillin resistant Staphylococcus aureus frequency in a tertiary care hospital, Lahore, Pakistan. Biomedica 25: 97-100.

Akhtar, N. 2010. Staphylococcal nasal carriage of health care workers. Journal of the College of Physicians and Surgeons Pakistan 20: 439-443.

Boyce, J.M., G. Potter-Bynoe, C. Chenevert and T. King. 1997. Environmental contamination due to methicillinresistant Staphylococcus aureus: possible infection control implications. Infection Control and Hospital Epidemiology 18: 622-627.

Cooper, B.S., S.P. Stone, C.C. Kibbler, B.D. Cookson, J.A. Roberts, G.F. Medley, G. Duckworth, R. Lai and S. Ebrahim. 2004. Isolation measures in the hospital management of methicillin-resistant Staphylococcus aureus (MRSA): Systematic review of literature. Journal of BMJ 329: 533-538.

Diekema, D. J., M.A. Pfaller, F.J. Schmitz, J. Smayovsky, J. Bell, R.N. Jones and M. Beach. 2001. Survey of infections due to Staphylococcus Species: Frequency of Occurrence and Antimicrobial susceptibility of isolates collected in the United States, Canada, Latin America, Europe, and the Western Pacific region for the SENTRY Antimicrobial surveillance program, 19971999. Clinical Infectious Diseases 32: 114-132.

Fluit, A.C., C.L. Wielders, J.F. Verhoef and F.J. Schmitz. 2001. Epidemiology and susceptibility of 3,051 Staphylococcus aureus isolates from 25 university hospitals participating in the European SENTRY Study. Journal of Clinical Microbiology 39: 3727-3732.

Friendship, B. and S. Weese. 2009. Methicillin resistant Staphylococcus aureus. Advances in pork production 20: $173-180$.

Gordon, J. 1993. Clinical significance of methicillin sensitive and methicilin resistant Staphlococcus in UK Hospitals and the relevance of povidone iodine in their control. Journal of Postgradaute Medicine 69: 106116.

Gould, I.M. 2005. The clinical significance of methicillin resistant Staphylococcus aureus. Journal of Hospital Infection 61: 277-282.

Hiramatsu, K., L. Cui, M. Kuroda and T. Ito. 2001. The emergence and evolution of methicillin resistant Staphylococcus aureus. Trends in Microbiology 9: 486-493.

Khanal, L.K. and B.K. Jha. 2010. Prevalence of methicillin resistant Staphylococcus aureus among skin infection cases at a hospital in Chitwan, Nepal. Journal of Nepal Medical College 12: 224-228.
Kikuchi, K. 2003. Genetic basis of neonatal methicillin resistant Staphylococcus aureus in Japan. Pediatrics International 45: 223-229.

Klutytmans, J. 1998. Reduction of surgical site infections in major surgery by elimination of nasal carriage of Staphylococcus aureus. Journal of Hospital Infection 40: 25-29.

McCarthy, N.L., P.S. Sullivan, R. Gaynes and D. Rimland. 2010. Risk factors associated with methicillin resistance among Staphylococcus aureus Infections in veterans. Infection Control and Hospital Epidemiology 31: 36-41.

Mintjes-deGroot, A.J., C.A. Van Hassel, J.A. Kaan, R.P. Verkooyen and H.A. Verbrugh. 2000. Impact of hospital-wide surveillance on hospital-acquired infections in an acute care hospital in the Netherlands. Journal of Hospital Infections 46:36-42.

Onanuga, A., A.R. Oyi, B.O. Olayinka and J.A. Onaolapo. 2005. Prevalence of community associated multiresistant Staphylococcus aureus among healthy women in Abuja, Nigeria. African Journal of Biotechnology 4: 942-945.

Onanuga, A. and T.C. Temedie. 2011. Nasal carriage of multi-drug resistant Staphylococcus aureus in healthy inhabitants of Amassoma in Niger delta region of Nigeria. African Health Sciences 11: 176-181.

Pant, J. and S.K. Rai. 2007. Occurrence of Staphylococcus aureus in hospital environment and staff in teaching hospital in Kathmandu, Nepal. Journal of Nepal Association Medical Laboratory Science 8: 72-73.

Pittet, D. and R.P. Wenzel. 1995. Nosocomial bloodstream infections. Secular trends in rates, mortality, and contribution to total hospital deaths. Archives of Internal Medicine 155: 1177-1184.

Polyzou, A., A. Slavakis, S. Pournaras, A.N. Maniatis, D. Sofianov and A. Tsakris. 2001. Predominance of methicillin- resistant Staphylococcus aureus clone susceptible to erythromycin and several other non - blactam antibiotics in a Greek hospital. Journal of Antimicrobial Chemotherapy 48: 231-234.

Rai, S.K., N.R. Tuladhar and H.G. Shrestha. 1990. Methicillin resistant Staphylococcus aureus in a tertiary medical care center, Nepal. Indian Journal of Medical Microbiology 8: 108-109.

Rijal, K.R., N. Pahari, B.K. Shrestha, A.K. Nepal, B. Paudel, P. Mahato and N. Skalko-Basnet. 2008. Prevalence of methicillin-resistant Staphylococcus aureus in school children of Pokhara. Nepal Medical College Journal 10: 192-195.

Sanjana, R.K., R. Shah, N. Chaudhary and Y.I. Singh. 2010. Prevalence and antimicrobial susceptibility pattern of methicillin resistant Staphylococcus aureus in CMSteaching hospital: a preliminary report. Journal of College of Medical Sciences-Nepal 6: 1-6.

Shaffer J.G. and M. Golden. 1969. Hospital epidemiology. In: Davidsohn I editor. Todd-Sanford Clinical Diagnosis 
Reena K Mukhiya et al./Prevalence of methicillin-resistant.....

by Laboratory Methods (14th ed.). Philadelphia. London. Toronto: W.B. Saunders, pp. 1074-1088.

Shakya, B., S. Shrestha and T. Mitra. 2010. Nasal carriage rate of methicillin resistant Staphylococcus aureus among at National Medical College Teaching Hospital, Birgunj, Nepal. Nepal Medical College Journal 12: 26-29.

Shrestha, B., B. Pokhrel and T. Mohapatra. 2009. Phenotypic characterization of nosocomial isolates of Staphylococcus aureus with reference to MRSA. Journal of Infection in Developing Countries 3: 554-560.

Shrestha, B., B. Pokhrel and T. Mohapatra. 2009. Study of nosocomial isolates of Staphylococcus aureus with special reference to methicillin resistant Staphylococcus aureus in a tertiary care hospital in Nepal. Nepal Medical College Journal 11: 123-126.
Tejero, A., M.A. Gutiérrez, M.J. Aiquel, M. Brandago, C. González and M.T. Broussain. 1991. Nasal carriage of Staphylococcus aureus among personnel working in a teaching hospital. Enfermedades Infeccioses Microbiologia Clinica 9: 351-353.

Verma S., S. Joshi, V. Chitnis, N. Hemwani and D. Chitnis. 2000. Growing problem of methicillin resistant staphylocci: Indian Scenario. Indian Journal of Medical Science 54: 535-540.

Vidhani, S., P.L. Mehndiratta and M.D. Mathur. 2001. Study of methicillin resistant Staphylococcus aureus isolates from high risk patients. Journal of Indian Medical Microbiology 9: 13-16.

Zahoor S., C. Vaishnavi, S. Kaur and A.Bhatia. 2001. Isolation and methicillin resistance- a preliminary report. Journal of Nepal Medical Association 40: 236. 
Nepal Journal of Science and Technology Vol. 13, No. 2 (2012) 185-190 